Conf $950740--35$

UCRL-JC-120583

PREPRINT

\title{
Tension in the Tie-Down Chains of a Shipping Container for Hazardous Material
}

\author{
Ting-Yu Lo
}

This paper was prepared for submittal to

the 1995 PVP Conference,

The American Society of Mechanical Engineers,

Honolulu, Hawaii

July 23-27, 1995

March 17, 1995

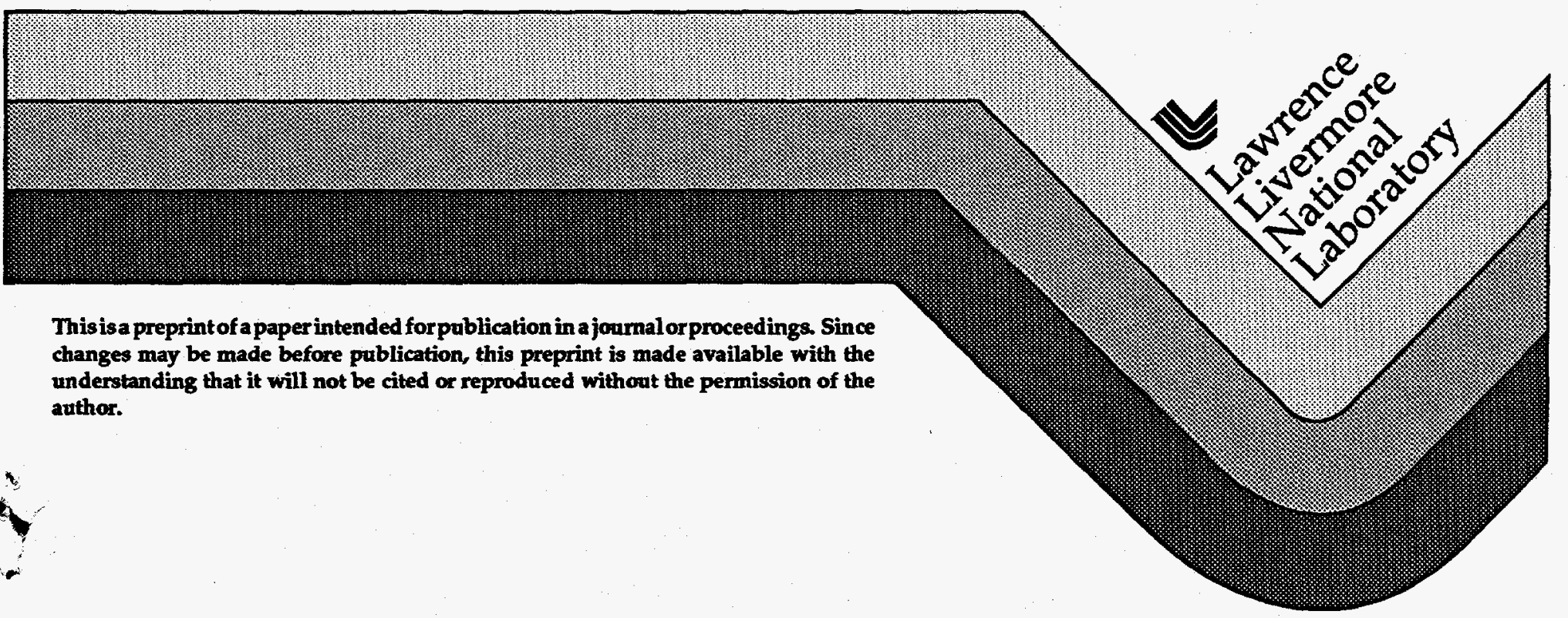




\section{DISCLAIMER}

This document was prepared as an account of work sponsored by an agency of the United States Government. Neither the United States Government nor the University of California nor any of their employees, makes any warranty, express or implied, or assumes any legal liability or responsibility for the accuracy, completeness, or usefulness of any information, apparatus, product, or process disclosed, or represents that its use would not infringe privately owned rights. Reference herein to any specific commercial product, process, or service by trade name, trademark, manufacturer, or otherwise, does not necessarily constitute or imply its endorsement, recommendation, or favoring by the United States Government or the University of California. The views and opinions of authors expressed herein do not necessarily state or reflect those of the United States Government or the University of California, and shall not be used for advertising or product endorsement purposes. 


\section{DISCLAIMER}

Portions of this document may be illegible in electronic image products. Images are produced from the best available original document. 


\title{
TENSION IN THE TIE-DOWN CHAINS OF A SHIPPING CONTAINER FOR HAZARDOUS MATERIAL
}

\author{
Ting-Yu Lo \\ Lawrence Livermore National Laboratory \\ Livermore, California
}

\begin{abstract}
Chains are frequently used to tie the shipping containers of hazardous material to a truck bed. The tie-down system is nonlinear when the container is subjected to a triaxial force during transit. It is nonlinear because chains cannot cany cumpressive force, and the base of the container may partially lift off from the truck bed. A method was developed to calculate the amount of tension in the chains. This methodology includes three assumptions: (1) No friction exists between the container and the truck bed; (2) The container and the truck bed are rigid; and (3) All chains are properly tightened (i.e., no slacks) during preparation for shipment The methodology employs an iterative process of a linear tie-down system. This linear system is derived from the nonlinear system with two additional assumptions: (a) All chains can carry compression as well as tension; and (b) There is a point contact between the container and the truck bed. This linear system has a closed-fam solution. After the first solution of the linear system is obtained, the unreascnable, or physically impossible, rotational degree of freedom of the container and the chains with compressive force are eliminated in the followup iterative calculations using the same linear system. Unreascnable rotation is detected when the results indicate a rotation of the container into the truck bed. Zero rotatioulal value is assigned to this degree of freedam in the follow-up iterations. For chains under compression, the chain length is replaced with a fictitiously large value because a long chain carries essentially no load. This process usually needs only two or three iterations and can easily be carried out in a spread sheet using such programs as Microsoft Excel $\alpha$ LOTUS 123.
\end{abstract}

\subsection{INTRODUCTION}

Chains are frequently used to tie a container of hazardous material to the truck bed of a transparter. Besides the chains, a tie-down system may include other components such as the brackets or shackles of a container, tensioners, tie-down adapters (or floor lugs), and tie-down tracks on the truck bed. For the containers designed in accordance with Title 10 of Code of Federal Regulations, Part 71 (10 CFR 71), it is required that tie-down devices which are structural parts of a package, must be capable of withstanding, without generating stress in any material of the package in excess of its yield strength, a static force applied to the center of gravity (cg) of the container and its contents. This static force should have a vertical component of two times the weight of the container and its contents, a horizontal component along the direction in which the vehicle travels of 10 times the weight, and a horizontal transverse component of five times the weight. The tie-down system needs to be designed to carry these loads.

\subsection{OBJECTIVE}

The objective of this study was to develop a methodology to calculate the tension force in the chains of a given tie-down system. By using trial-and-error and this methodology, it is then possible to obtain an appropriate layout (the number of chains and the length and crientation of each chain) for a tit-down system.

\subsection{METHOD OF ANALYSIS}

A tie-down system imviving chains is nonlinear when the container is subjected to a triaxial force during transit. If is nonlinear because chains cannot carry compressive force, and the base of the container may lift off partially from the tucik bed. A methodology was developed to calculate the tension force in the chains of a given tiedown system. This methodology inchudes the following assumptions:

(1) No friction exists between the container and the truck bed; 
(2) Except the chains, all components including the container and the truck bed are rigid; and

(3) All chains are properly tightened during preparation for shipment. Slack in chains is not considered in this methodology. The small preload in chains due to tightening is negligible compared to the chain force proctuced by the static force discussed in Section 1.0.

The methodology employs an iterative process and a linearized tiedown system. This linear system is derived fram the nonlinear system with two additional assurmptions: (a) All chains can carry compression as well as tension; and (b) The contact condition between the truck bed and the support system of the container is known. This given contact condition can be ane of the following three: (1) a point contact; (2) a line or a two-point contact on one side of the support, and (3) a line $\alpha$ a two-point contact along the front edge of the suppart. This linear system has a closed-form solution. The mathematical fomulation of this linear system is presented in Section 3.1. After the first iteration of the linear system, unreascanable a physically impossible rotational degree of freedom of the container is eliminated in the follow-up iterarive calculations. Unreasconable rotation is detected when the results indicate a rotation of the container into the truck bed. This rotational degree of freedom is then constrained in the follow-up iterations. For chains under compression, the chain length is replaced with a fictitiously large value because long chain carries essentially no load. This iterative process can easily be carried out in a spread sheet using such programs as Microsoft Excel or LOTUS 123. Detailed discussion of this iterative process is presented in Section 3.2.

\subsection{A Linear The-Dewn System}

Figure 1 shows two kinds of cocrdinate systems used in this methodology for a container and its tie-down system. The global coardinate system $(X, Y$, and $Z)$ is applicable to the container. The arigin of this coardinate system can be located anywhere at the discretion of the analyst. In Fig 1, the crigin is located at Point $\mathbf{O}$, which is at the left front camer of the suppart. The local coardinate system is for the chains. Each chain has its own local coordinate system. The crigin of each of these local coordinate systems is located at the anchor point of the chain on the truck bed. These local cocrinate systems are parallel to the global coordinate system. A typical local coardinate system $(x, y$, and $z$ ) for the chain comected to Point $B$ (Chain 5) is also shown in Fig. 1. The total number of chains and the length and crientation of these chains are assumed to be known for the given tie-down system.

\subsubsection{A Typical Chain.}

For a typical chain (for example, Chain 5 comected to Point B) we have the following:

Chain length, $s=\sqrt{x^{2}+y^{2}+z^{2}}-c$.

Elongation of the chain, $d_{z}=\left(x d_{x}+y d_{y}+z d_{z}\right) /(s+c)$

Spring constant, $k=n a / s$.

Force in the chain,

$F=k d_{s}=n a\left(x d_{x}+y d_{y}+z d_{z}\right) /[s(s+c)]$

In the above equations,

$c=$ the length of the rigid tensioner for the chain,

$n=$ actual number of chains used for the chain under consideration,

$a=a$ chain property depending on the size and material properties of the chain,

$d_{i}=$ displacement in direction " $i$ " of Point B (" $z$ " can be $x, y$, or z) relative to the truck bed, and

$(x, y, z)=$ coordinates of Point B in the local coordinate system.

Throughout this calculation, it is not necessary to know the exact value of " $a$ " because it is canceled out in the calculation of chain forces if the same type (size and material) of chains is used throughout the tie-down system. If the displacement of the container relative to the truck bed or the elongation of a chain is needed, the actual value of " $a$ " is then required.

For a given displacement vector $\left(d_{x}, d_{y}, d_{z} d_{x x}, d_{y,}, d_{z z}\right)$ of the rigid container at Point $O$, the displacement vector of Point $B$ can be expressed as functions of $d_{x}, d_{y}, d_{z}, d_{x x}, d_{y}$, and $d_{z z}$.

$$
\left\{\begin{array}{l}
d_{x} \\
d_{y} \\
d_{z}
\end{array}\right\}=\left[\begin{array}{cccccc}
1 & 0 & 0 & 0 & Z & -Y \\
0 & 1 & 0 & -Z & 0 & X \\
0 & 0 & 1 & Y & -X & 0
\end{array}\right]\left\{\begin{array}{l}
d_{X} \\
d_{Y} \\
d_{Z} \\
d_{X X} \\
d_{Y Y} \\
d_{Z Z}
\end{array}\right\}
$$

where $X, Y$, and $Z$ are the coordinates of Point $B$, the typical point of the container, in the global coordinate system.

The components of the chain force $F$ in the $x, y$, and $z$ (or $X, Y$, and 2 ) directions are as follows:

$\left\{\begin{array}{l}F_{X} \\ F_{y} \\ F_{z}\end{array}\right\}=\left\{\begin{array}{l}F_{X} \\ F_{Y} \\ F_{Z}\end{array}\right\}=\frac{F}{s+c}\left\{\begin{array}{l}x \\ y \\ z\end{array}\right\}$,

where $x, y$, and $z$ are the coordinates of Point $B$ in the local coordinate system. 
The moments about the $X, Y$, and $Z$ axes due to the chain force $F$ acting on the container at Point $B$ are

$$
\left\{\begin{array}{l}
M_{x o} \\
M_{y o} \\
M_{z 0}
\end{array}\right\}=\left\{\begin{array}{l}
M_{x O} \\
M_{y o} \\
M_{z O}
\end{array}\right\}=\left[\begin{array}{ccc}
0 & -Z & Y \\
Z & 0 & -X \\
-Y & X & 0
\end{array}\right]\left\{\begin{array}{l}
F_{x} \\
F_{y} \\
F_{z}
\end{array}\right\} .
$$

The above formulas for the chain connected to Point B are applicable to other chains of the tie-down system.

\section{1 .2 Equillbrium of the Container}

\subsubsection{Equations of Equilibrium}

Under the external static force shown in Fig. 1, the equilibrium equations for the container considering the forces in all of the chains are as follows:

$$
\sum_{i=1}^{m}\left(F_{x}\right)_{i}=10 W
$$

$$
\sum_{i=1}^{m}\left(F_{y}\right)_{i}=5 W
$$

$$
\sum_{i=1}^{m}\left(F_{z}\right)_{i}=2 W+R_{0}+R_{1}+R_{2}
$$

$$
\sum_{i=1}^{m}\left(M_{x}\right)_{i}=2 W Y_{c g}-5 W Z_{c g}+R_{2} Y_{2}
$$$$
\sum_{i=1}^{m}\left(M_{y}\right)_{i}=10 W Z_{c i}-2 W X_{c g}-R_{1} X_{1}
$$$$
\sum_{i=1}^{m}\left(M_{z}\right)_{i}=5 W X_{c g}-10 W Y_{c g}
$$

where

$W=$ Total weight of the container and its contents,

$R_{0}=$ Reaction force of truck bed at Point $O$,

$R_{I}=$ Reaction force of truck bed at left rear comer of the support,

$R_{2}=$ Reaction force of truck bed at right front comer of the support,

$m=$ total number of chains,

$\left(X_{C g}, Y_{C g}, Z_{C g}\right)=$ coordinates of the CG of the container and its contents,

$\left(X_{1}, 0.0,0.0\right)=$ coordinates of the reaction force $R_{1}$ in global coordinate system, $\left(0.0, Y_{2}, 0.0\right)=$ coordinates of the reaction force $R_{2}$ in global coordinate system.

The above equilibrium equations are for a static load with $10 \mathrm{~g}$ longitudinal, $5 \mathrm{~g}$ lateral, and $2 \mathrm{~g}$ vertical components. Similar equations can be obtained for other loading conditions if necessary.

\subsubsection{Loading and Boundary Conditions}

The boundary conditions for four loading cases are as follows:

Case 1 - 10g Forward Load

$$
d_{y}=d_{z}=d_{x x}=d_{z}=0 .
$$

Case 2 - 5g Lateral Load

$$
d_{z}=d_{\boldsymbol{r}}=0
$$

Case 3 - 2g Vertical Load

$$
R_{0}=R_{1}=R_{2}=0
$$

Case 4 - $10 \mathrm{~g}$ Forward, $5 \mathrm{~g}$ Lateral, and $2 \mathrm{~g}$ Vertical Load

$d_{z}=0$

If the container and truck bed have more than one point of contact, one of the following two boundary conditions is also applicable.

$$
\begin{aligned}
& d_{x x}=0 \quad\left(\text { if } R_{1}=0\right), \text { and } \\
& \left.d_{Y Y}=0 \quad \text { (if } R_{2}=0\right)
\end{aligned}
$$

\subsection{Iterative Process in Solvina the Equations of} Equilibrium

Spread sheet programs such as LOUTS 123 or Microsoft Excel may be used to perform calculations in accordance with the equations presented in Section 3.1. In those equations, the variables are $d_{x} d_{y}, d_{z} d_{x x}, d_{y}, d_{z z}, R_{0}, R_{1}$, and $R_{2}$. Straightforwand elimination of variable method may be used to solve these equations. For loading and boundary conditions 1, 2 and 3 , the solution can be obtained easily because many variables are zero and it is very clear which of the variables, $R_{l}$ or $R_{2}$, is zero. Chains in compression can be eliminated by assigning fictitiously long chain length because a long chain will have small stiffness and, therefore, carry little load. The iterative process used to eliminate the chains with compressive force is straightforwand and can be done easily on the spread sheet.

For loading and boundary condition 4 , the iterative process is more complicated becamse it is not clear at the outset which of the variables $R_{1}$ and $R_{2}$ will be active because the container may rotate about any of the two horizontal axes. It may also pivot at Point $O$. The first step in this iterative process is to assume that both $R_{1}$ and $R_{2}$ are equal to zero (that is $d_{x x} \neq 0$ and $d_{y y} \neq 0$ ) and 
there is only one point of contact between the container and the truck bed. The results of this iteration will provide the necessary clue becamse either $d_{x x}$ or $d_{y r}$ will most likely be unrealistic due to the limitation that the container simply cannot rotate into the truck bed. In this initial iteration, the other type of iteration required to eliminate chains with compressive force can also be carried out. Once it is known which of $d x x$ and $d x$ is equal to zero, the same process, although with slightly different boundary conditions, can be repeated to obtain the final solution.

The analysis method described above was validated by a three dimensional finite element analysis of a sample problem using NIKE3D code, which is available at the Lawrence Livermore National Laboratory. Close agreement was observed between spread sheet results using the analysis methodology described above and the finite element analysis results.

\subsection{A SAMPLE ANALYSIS}

The tie-down system of a sample shipping container is shown in Fig. 1. This system has 20 chains and was the result of several trial-and-error studies using the spread sheet calculations described above. It started with 24 chains. The tie-down system is symmetrical about a vertical plane through the longitudinal axis of the container. The shackles or brackets are identified with letters $A$ through $J$ and the chains are numbered from 1 to 12 on each side of the container. Only the chains on the righthand-side (RHS) of the container are shown in Fig. 1. Chains 1 and 12 on both sides were eliminated during the trial-and-error studies and are also not shown in the figure. Chains 6 and 11 inciude two actual chains each. Please note that each of brackets $A, E, F$, and $J$ include two actual brackets. One is in the front and the other is in the back of the support cradles.

The analysis results for Case 4 loading and boundary conditions are presented in Table 1. The forces and force components in the chains are shown on the left hand side (LHS) of each table; the forces and force components on the brackets or shackles are shown on the right hand side. The total truck bed reaction force is provided at the bottom of these tables. Most of the forces are presented as multiples of the total weight (W) of the container and its contents. The container shown in Fig. 1 has a total weight of 3000 pounds. The columns under $d_{x}, d_{y}, d_{2} d_{x x}, d_{r}, d_{z z}$ headings show the axial forces in the chains due to the effect of individual displacement component of container in the $X, Y$, and $Z$ directions and rotations about $X, Y$, and $Z$ axes in the global coordinate system.

Under Case 4 loading conditions, the container rotates about the $Y$ axis, i.e., $d x x=0$, which was found during the iteration described in Section 3.2. For Chains 2, 4, 7 and 9, which are comnected to lower elevation brackets, the tension is due mainly to container translation $(d x)$ in the longitudinal direction. The tension force in chains 6 and 11 on the RHS is due to the combined effect of translation $d_{y}$ and torsion $d_{z z}$. Chains 6 and 11 on the LHS have zero tension force due to the $5 \mathrm{~g}$ lateral load. For chains (Chains 5 and 10) connected to higher elevation shackles of the container, their tension forces are mainly due to rotation $\left(d_{2 y}\right)$ of the container. The tension force in Chain 10 is lower than that of Chain 5 becamse Chain 10 is closer to the axis of motation. Chains 5 and $\mathbf{1 0}$ on the RHS have larger tension force than those on the LHS due to the translational component $d_{r}$ of the container. Chain 4 carries more load than Chain 2 because it is shorter and therefore stiffer. The same is true for Chains 7 and 9. Chains 3 and 8 are in front of the container and, therefore, carry no load or very small load.

\subsection{SUMMARY AND CONCLUSIONS}

A methodology was developed to calculate the tension force in the chains of a tie-down system of a shipping container. This methodology employs an iterative process and a linearized tiedown system. The spread sheet program LOTUS 123 was used to implement this methodology. Sensitivity studies can conveniently be carried out on this spread sheet to design the tiedown system of a shipping container. Because the effects of each of the container displacement components $\left(d_{x}, d_{r}, d z_{0} d_{x x}, d_{r}\right.$, and $d_{2 z}$ ) can be calculated, the effectiveness of each chain can easily be observed or identified. This capability helps the tiedown design tremendously. This methodology was applied to a sample shipping container with satisfactory result.

This work was performed under the auspices of the U.S. Department of Energy by Lawrence Livermore National Laboratory under contract no. W-7405-Eng-48. 


\section{TABLE 1 TENSION IN THE TIE-DOWN CHAINS OF A SAMPLE SHIPPING CONTAINER UNDER A $10 G$ LONGTUDINAL, 5G TRANSVERSE, AND 2G VERTICAL LOAD}

SUMMARY

Case HSXYZ - SST Configuration ( $d Z=d X X=0$ )

(File: HSXYZ4.wKS)

$(2 / 14 / 94)$

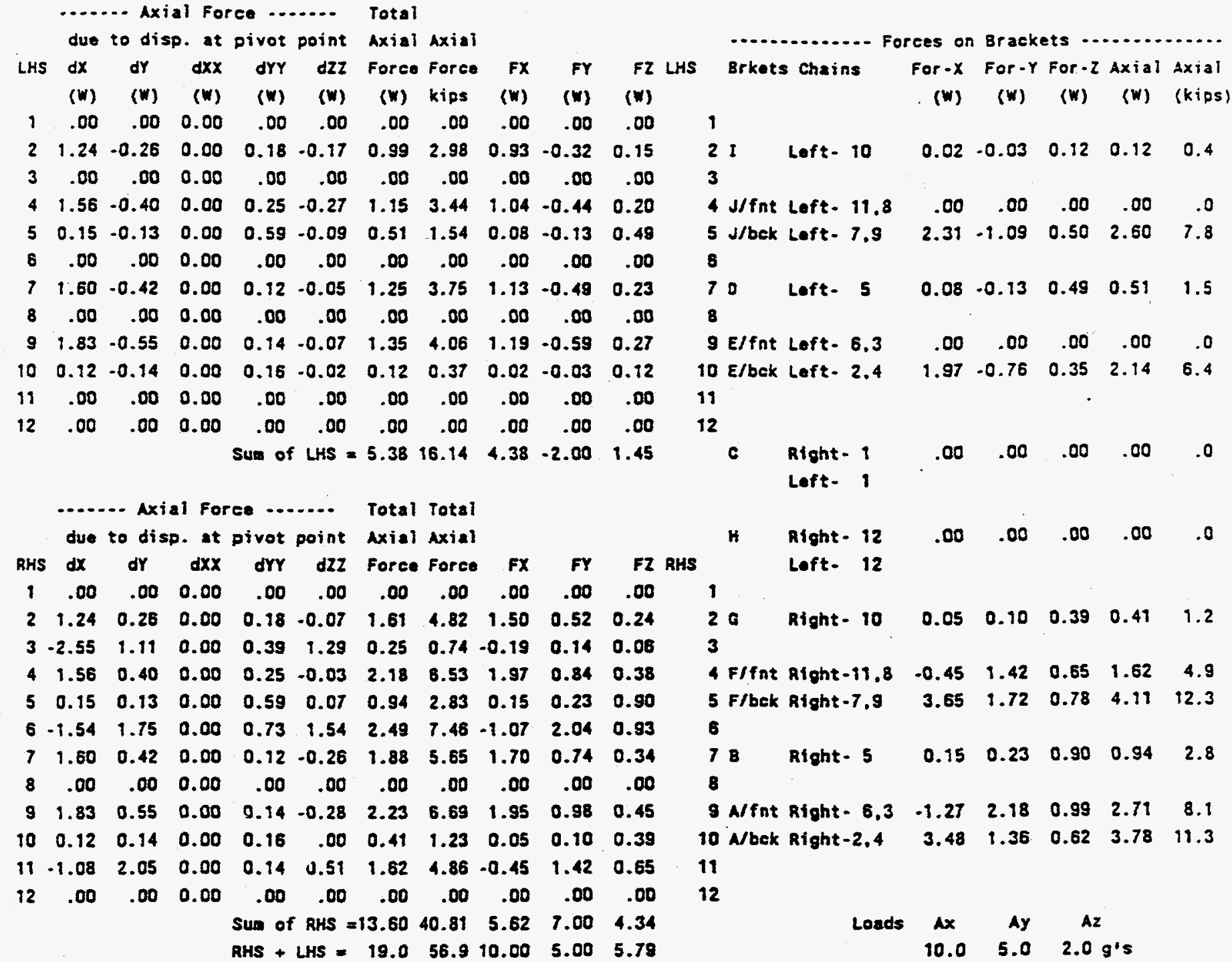

NOTE: Chains $\# 6$ and $\# 19$ include two chains each, wial force = half.

Truck sed Reaction force $=3.79 \mathrm{~W}=11.38$ kips 


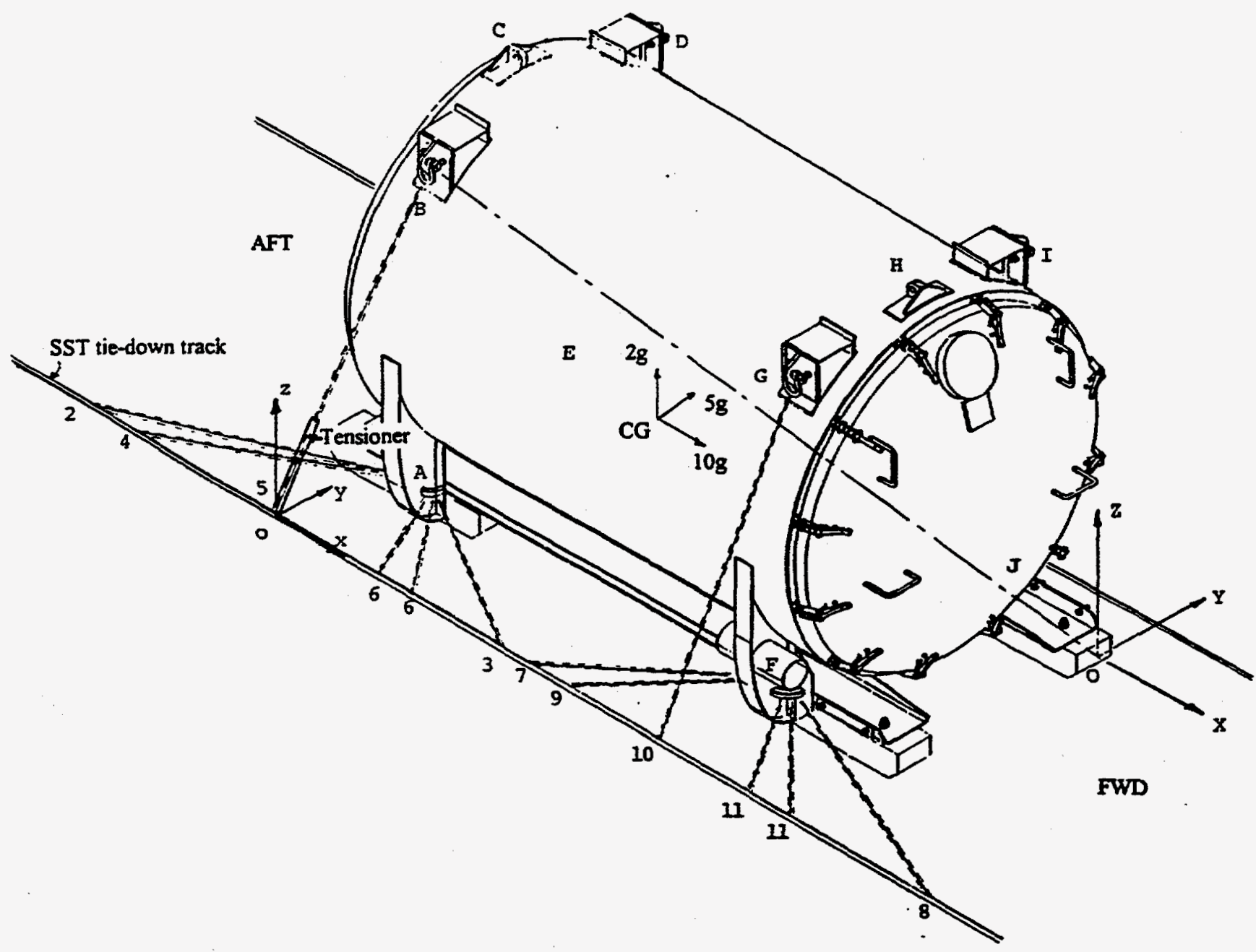

FIGURE 1 TIE-DOWN SYSTEM OF A SAMPLE SHIPPING CONTAINER 